\title{
The Image Steganography Method Based on the Nonlinear Optimization
}

\author{
Yu Chen, Ren-er Yang,Min Yuan, Jun Mo,Cheng-hong Ma \\ Faculty of Information Science and Technology, \\ Ningbo University, \\ Ningbo ,China \\ e-mail:a88330130@sina.com
}

\begin{abstract}
On digital image steganography, the traditional LSB method usually makes the histogram's change relatively large after the image steganography; therefore the hidden information can be detected easily. This paper puts forward a new method which based on the nonlinear optimization to determine the best LSB steganography technology of histogram detecting. It can make the histogram's total change to be as smaller as possible and difficult to be detected.
\end{abstract} LSB

Keywords-steganography; nonlinear optimization; improved

\section{INTRODUCTION}

With the popularization of Internet and the rapid development of multimedia and communication technology, it provides a great convenience for the application of information compression, storage, replication and transmission. Various confidential information which includes national security information, military information and private information is transferred by the Internet[1],[2]. However, the internet is open. It brings us convenience at the same time comes about many security problems, such as the copyright infringement of multimedia, the piracy distort of digital production and so on. People have paid more and more attention to the security of transmission information.

The optimization method and its theory come from the military affairs, management, economy and engineering field. The depth and scope of its content develop with the development of technology in different stages. During the two World Wars, especially the Second World War, people put forward many military optimization problems. These problems and their solutions had salient features: the data was real; the solvers came from different subjects; the processing method had the physical idea. After the Second World War, the application of optimization method turns to the civil problems; engineering technology and modern management are put forward. Especially in recent thirty years, the need of science and technology and the development of computer provide rich basis and methods for the optimization method and its theory. The application range of optimization is more and more widely, involves design, operation, industrial processes, and the analysis of providing device, the decision of economic function and so on. Because the optimization method is searching for the method which brings the best effect, it has very important practical significance.
The aim of digital steganography is to hide the communication information which is carried by the digital media. It use the data redundancy of digital medias and the physiological and mental characters of our human beings' organ perception to embed the secret information which is encoded or encrypted in public digital media in a special way.

The LSB embedding is the most common method of digital steganography. The traditional LSB method converts the secret information to binary data streams, and then replaces the lowest bit of grey level of the vector image's pixel. This method can hide a large quantity of data, and is difficult to be sensed. It's applied widely in practice. But it usually makes the change of grey level histogram relatively large and it can be detected easily. Thus we put forward a nonlinear optimization of LSB embedding method which can make the histogram's change to be the smallest. We denote $S_{k}$ as the grey level histogram's function[3],[4],[5]. For the grey image of 8 bits, $k \in[0,255]$. And $s_{k}$ is a vector which represents the number of image's pixel value, namely

$$
s_{k}=\overline{\overline{\left\{(i, j) \mid a_{i j}=k\right\}}}=\sum_{i=1}^{W} \sum_{j=1}^{H} \delta\left(k, a_{i j}\right) \text { (1), }
$$

where

$$
\delta(u, v)=\left\{\begin{array}{l}
1, \text { if } u=v \\
0, \text { if } u \neq v
\end{array}\right.
$$

And $a_{i j}$ means the image's pixel value at $(i, j), W$ is the image's width, $H$ is the image's height.

\section{StEgANOGRAPHY METHOD BASED ON THE NONLINEAR OPTIMIZATION}

To overcome the traditional LSB method's disadvantage, this paper provides an improved LSB embedding method which uses the nonlinear optimization to make the histogram's change to be the smallest.

In order to solve the model conveniently, we use a matrix $C$ that the size is $512 \times 512$ to replace the binary data stream which is converted from the secret information. The producing way of matrix $C$ is as follows: we first give a matrix $q$ of $512 \times 512$ which obeys the uniform distribution. Its domain is $[0,1]$ and the mean is 0.5 . 
And $q=\left(\begin{array}{ccc}q_{(1,1)} & \cdots & q_{(1,512)} \\ \vdots & \ddots & \vdots \\ q_{(512,1)} & \cdots & q_{(512,512)}\end{array}\right)$. If $q_{i j}>0.5$, then the value of $C_{i j}$ is 1 , or else is 0 .

The generation of the binary data stream's transformation matrix $C$ : convert the secret information which is needed to be embedded into binary data stream matrix $C$. Assume $c_{i j}$ represents the data of the pixel's terminal position which is inserted at the $(i, j)$. And it equals 0 or 1 . We have

$$
C=\left(\begin{array}{ccc}
c_{11} & \cdots & c_{1 n} \\
\vdots & \ddots & \vdots \\
c_{n 1} & \cdots & c_{n n}
\end{array}\right)
$$

The generation of the pixel grey level's lowest bit $S$ : extracting the pixel grey level's lowest bit matrix $S$ of the image G. $S_{i j}$ represents the value of the pixel's terminal position at the $(i, j)$. It equals 0 or 1 . We denote the spatial domain of the image as $a, a_{i j}$ represents the pixel's value. The range of $a_{i j}$ is $(0,255)$. We have $_{S}=\left(\begin{array}{ccc}s_{11} & \ldots & s_{1 n} \\ \vdots & \ddots & \vdots \\ s_{n 1} & \cdots & s_{n n}\end{array}\right), a=\left(\begin{array}{ccc}a_{11} & \ldots & a_{1 n} \\ \vdots & \ddots & \vdots \\ a_{n 1} & \cdots & a_{n n}\end{array}\right)$.

The operation of embedding the information directly: replacing $C$ by the pixel's lowest bit $S$ of the corresponding image directly; then we can get a new image $A_{1}$, and a transformation matrix $b$, where

$$
b_{i j}=f\left(s_{i j}, c_{i j}\right)
$$

The definition of $f$ as follows: for given $i=i_{0}, j=j_{0}$, if $s_{i_{0} j_{0}}=c_{i_{0} j_{0}}$, then $b_{i_{0} j_{0}}=0$, or else $b_{i_{0} j_{0}}=1$. So we can deem that if the value of pixel's terminal position $S_{i j}$ equals the value of $c_{i j}$, the pixel's value of the original image at some point is not need to change and represented as 0 . Otherwise it needs to be changed and the change is 1 or -1 . Therefore we only need to do a $512 \times 512$ cycle and get a matrix $b$. And $b=\left(\begin{array}{ccc}b_{11} & \cdots & b_{1 n} \\ \vdots & \ddots & \vdots \\ b_{n 1} & \cdots & b_{n n}\end{array}\right)$.

The operation of $b$ 's statistics which is about the pixel: converting the matrix $b$ into the count vector $h$, $h=\left(h_{0}, \cdots, h_{k}, \cdots, h_{m}\right)^{T}$ and $m=255$

$$
h_{k}=\overline{\overline{\left\{(\mathrm{i}, \mathrm{j}) \mid \mathrm{b}_{i j}=1, \mathrm{a}_{i j}=\mathrm{k}\right\}}}
$$

means we classify $a_{i j}$ which corresponds to $b_{i j}=1$ as $0 \sim 255$. That's for $b_{i j}=1$ and $a_{i j}=0$ belongs to the same category, for $b_{i j}=1$ and $a_{i j}=1$ belongs to another category, and so forth. Thus we can get 256 categories and $h_{k}$ is the number of every category.

The optimization: $h_{k}$ represents

$b_{i j}=1$ and $a_{i j}=k$. That means the original pixel is $k$, they need to be changed to $k-1$ or $k+1$ and their number is

$h_{k}^{(1)} \quad, \quad h_{k}^{(2)} \quad, \quad h^{(1)}=\left(h_{0}^{(1)}, \cdots, h_{k}^{(1)}, \cdots, h_{m}^{(1)}\right)^{T}$, $h^{(2)}=\left(h_{0}^{(2)}, \cdots, h_{k}^{(2)}, \cdots, h_{m}^{(2)}\right)^{T} \quad$. For $\quad k=0$ and $k=255$, we have $h_{0}^{(1)}=0, h_{255}^{(2)}=0$. So we denote the new count which is changed as $h_{k}^{\prime}$, $h^{\prime}=\left(h_{0}^{\prime}, \cdots, h_{k}^{\prime}, \cdots, h_{m}^{\prime}\right)^{T}$. And we consider the sum of the absolute value of the original count and new count's difference as the objective function of the optimization. Here is the unconditional optimization model. Its mathematical expression is

$$
\begin{aligned}
& \min \sum_{k=0}^{255}\left|h_{k}-h_{k}^{\prime}\right| \\
& \left\{\begin{array}{c}
h_{k}^{(1)}+h_{k}^{(2)}=h_{k}, \\
h_{k+1}^{(1)}+h_{k-1}^{(2)}=h_{k}^{\prime}, \\
h_{255}^{(2)}=0, h_{0}^{(1)}=0,
\end{array}\right.
\end{aligned}
$$

Using the result of the above optimization gets the new embedding image: from the optimization, we can get $h^{(1)}, h^{(2)}$, then we scan the original image and change it , therefore we can get the stego image that the grey level histogram's change is the smallest.

From the above, we can get the new image that the grey level histogram's change is the smallest after embedding the secret information. And it not easy to be detected that it weather inserted the secret information by the attacker.

\section{PERFORMANCE}

Firstly, we select four images randomly as shown in figure 1. By our method and using the software Lingo, we can get the optimal solution $h^{(1)}, h^{(2)}$. Here we not list them.

Secondly, we use the formula of PSNR to get the PSNR of the image $A$ and $A^{\prime}$.

$$
P S N R=10 \times \log \left(\frac{255^{2}}{M S E}\right)
$$




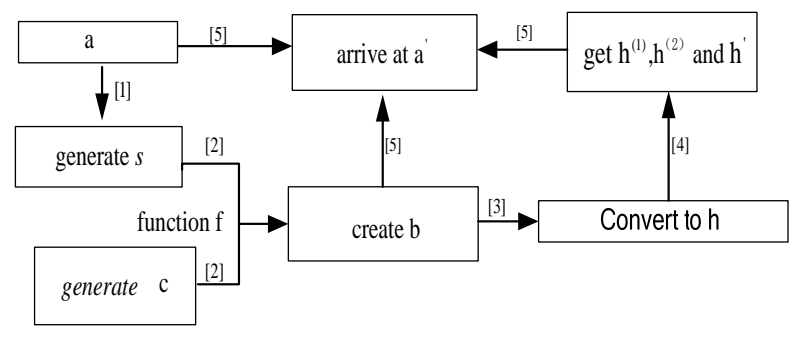

Figure 1. performance

$$
M S E=\frac{\sum_{i=1}^{512} \sum_{j=1}^{512}\left(a_{i j}-a_{i j}^{\prime}\right)}{512^{2}}
$$

Theoretically, their PSRN should be approximate. Then we get the $A^{\prime}, A^{\prime \prime}$ and $A$ 's histogram $s^{(1)}, s^{(2)}$ and $s$. Lastly we get the histogram of the difference of the $s^{(1)}, S^{(2)}$ and $s$. The flow chart of the above is as shown in figure 2 .

The following table gives the PSRN of the four images' secret information by the optimization and by inserting directly. It also gives the change number of the histogram.

From the table 1, we can firstly know this invention puts forward that using the nonlinear optimization to solve the steganography can reduce the histogram's change number on a large scale which by the traditional method. Secondly, for the same image, their PSRN is the same and it closes to 51. Thirdly, for different images, their histogram's change number is also very different by using the above two methods.

And we find for the image $\mathrm{C}$, the histogram's change number by using the optimization reduces to $5.7 \%$ compared with inserts directly, it's a perfect result and has a big difference with other. We can believe that the image C is very perfect to this data stream.

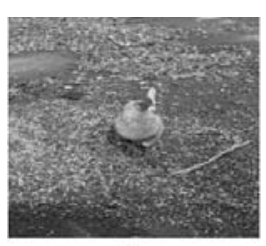

A

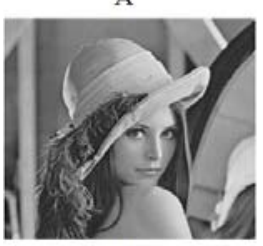

C

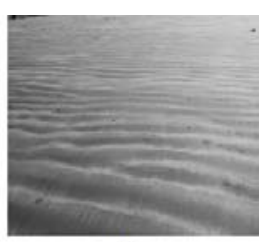

B

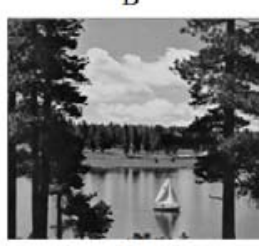

D.
Figure 2.the original images
In order to illustrate the difference by the data, we give the change of the images' grey level histogram by using the optimization and inserting directly. It's shown in figure 3. From the difference graph of the histogram by optimization and inserting directly, the image $C$ is better than the image A, B and D. For all the four images, their difference graph's rag of the histogram by using the optimization is less than by inserting directly, and is obviously better than the difference graph of the optimized histogram and directly inserted histogram.

\section{CONCLUSIONS}

In this paper, we use the nonlinear optimization to realize the LSB steganography. Compared with the direct way of insert, it makes the histogram's change rather small. So by the standard of histogram, the detecting method is hard to be successful. In the future's study, we'll consider more complex environment and introduce more practical factors, for example, the signal transmission noise. In addiction, the nonlinear optimization makes the histogram's change come to the smallest at the same time it usually sacrifices the load time.

\section{ACKNOWLEDGMENT}

Project is supported by This work was supported in part by the National Science and Technology Major Project (No. 2011ZX03002-004-02), the Program for New Century Excellent Talents in University (No. NCET-08-0706), the Science Foundation of Zhejiang Province (No. R1110416), the Specialized Research Fund for the Doctoral Program of Higher Education (No. 20113305110002), the Program for Technology Innovation Team of Ningbo Government(No. 2011B81002).Zhejiang Scientific and Technical Key Innovation Team of New Generation Mobile, Internet Client Software (No.2010E10005).

\section{REFERENCES}

[1] H.Wang and S.Wang, “Cyber warfare: Steganography vs. steganalysis ,”Commun. ACM, vol. 47, no. 10, pp. 76-82, 2004.

[2] J. Fridrich and M. Goljan, "Practical steganalysis of digital im-agesstate of the art," in Proc. SPIE Security Watermarking Multi-media Contents IV, , vol. 4675, San Jose, CA, Jan. 2002, pp. 1-13.

[3] P.Sallee,"Model-Based Steganography," in Proceedings of the 6th Information Hiding Workshop, Lecture Notes in Computer Science,2939, Springer-Verlag, pp. 154-167, 2004.

[4] X. Zhang, S. Wang, and K. Zhang, "Steganography with Least Histogram Abnormality,” Computer Network Security, Lecture Notes in Computer Science, 2776, pp. 395-406, 2003.

[5] H. Wu, J. Dugelay, and Y. Cheung, "A Data Mapping Method for Steganography and Its Application to Images," in Proceedings of the 10th Information Hiding Workshop, Lecture Notes in Computer Science, 5284, pp. 236-250, 2008.

[6] J. Harmsen, W. Pearlman., "Higher-order statistical steganalysis of palette images," in Security and Watermarking of Multimedia Contents , Proc. SPIE 5020, pp. 131-142, 2003. 
TABLE 1 THE PSRN OF FOUR IMAGES AND THE CHANGE NUMBER OF THE HISTOGRAM

\begin{tabular}{|c|c|c|c|c|c|}
\hline \multirow[t]{2}{*}{ Image } & \multicolumn{2}{|c|}{ Method1:inserting directly } & \multicolumn{2}{|c|}{ Method 2 : optimization } & \multirow[b]{2}{*}{$\Delta_{2} / \Delta_{1} \times 100 \%$} \\
\hline & $\begin{array}{c}\text { The change number } \\
\text { of histogram } \Delta_{1}\end{array}$ & PSNR & $\begin{array}{c}\text { The change } \\
\text { number of } \\
\text { histogram } \Delta_{2}\end{array}$ & PSNR & \\
\hline A & 6014 & 51.15 & 1330 & 51.15 & 22.12 \\
\hline $\mathrm{B}$ & 6012 & 51.14 & 1232 & 51.14 & 20.49 \\
\hline $\mathrm{C}$ & 8262 & 51.14 & 294 & 51.14 & 5.749 \\
\hline $\mathrm{D}$ & 21392 & 51.13 & 4944 & 51.13 & 23.11 \\
\hline
\end{tabular}
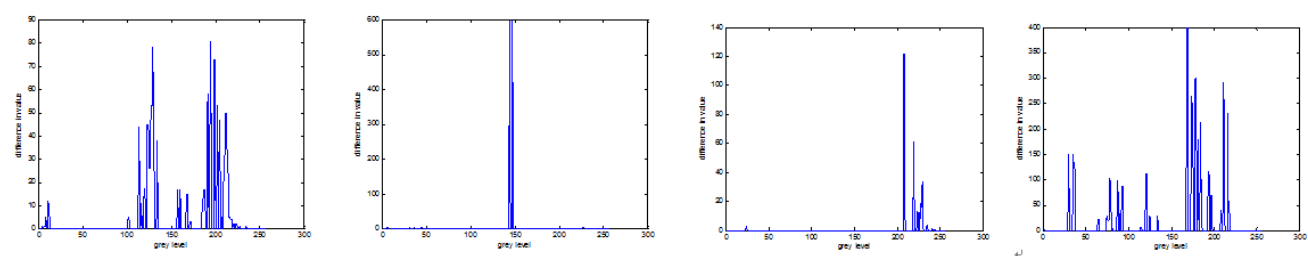

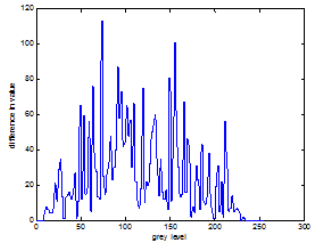

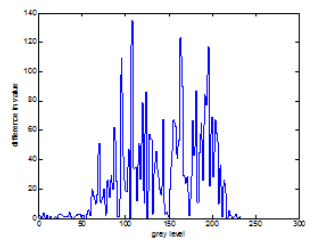

B

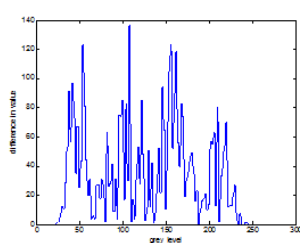

C

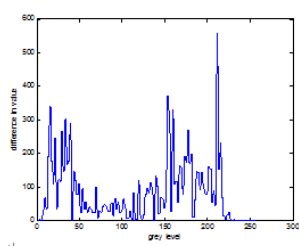

D

Figure 3. the difference between two methods 